\title{
BA-ELISA isolation of cytosolic Salmonella and vacuolar Salmonella
}

\author{
Suke Liu ${ }^{1 *}$ \\ ${ }^{1}$ department of Biotechnology and Food Science, Tianjin University of Commerce, Tianjin 300134, China;
}

\begin{abstract}
Salmonella is a pathogenic Gram-negative bacterium and important zoonotic pathogen survive in host cell, which is well known to cause gastrointestinal infections and even death in human through food contamination. Salmonella survives and reproduces within cells in two forms of cytosolic Salmonella and vacuolar Salmonella. However, the current researches on the mechanism of Salmonella infection was mostly based on the total numbers, and there is no clear distinction between cytosolic Salmonella and vacuolar Salmonella. This article combined biotin-streptavidin-amplified enzyme-linked immunosorbent assay(BAELISA) and Flat colony counting method to separate and quantify cytosolic Salmonella and vacuolar Salmonella. The results showed that the anti-Salmonella antibody with a dilution ratio of 1:500 can bind $\sim 1700$ Salmonella, and the binding rate reached to $70 \%$. In terms of bacterial counts, vacuolar Salmonella dominates in the early stage of Salmonella infection, and cytosolic Salmonella dominates in the later stage, the reproduction rate of vacuolar Salmonella is significantly lower than cytosolic Salmonella during Salmonella infection, the dynamic growth curve of cytosolic Salmonella and vacuolar Salmonella intuitively reflected the proliferation status of the two forms of Salmonella during the entire process of Salmonella infection.
\end{abstract}

\section{Introduction}

Salmonella, belonging to Gram-negative facultative anaerobes, is one of the important pathogenic bacteria causing food poisoning and the most studied human pathogen ${ }^{[1]}$. The pathogenicity of Salmonella depends on its ability to survive in nonphagocytic epithelial cells ${ }^{[2]}$. There are two ways of Salmonella surviving in the epithelium. One is surviving within the Salmonella containing vacuole (SCV) ${ }^{[3]}$ and the other is for escaping from within the membrane compromised SCV to acquire abundant nutrients in the cytoplasm and propagating at a faster rate for Salmonella ${ }^{[4]}$. At present, much of the researches about the propagation mechanisms of intracellular Salmonella are still at the level of total bacterial counts, and the distinction between Salmonella within the SCV and free Salmonella within the host cytoplasm has been in an ambiguous area. Experimenters have used fluorescently labeled Salmonella to infect macrophages for fluorescent screening and enrichment of intracellular pathogens by flow cytometry, but the shearing forces caused by flow cytometric sorting can lead to serious damage to the pathogens ${ }^{[5]}$. by paramagnetic nanoparticle labelled Salmonella to infect macrophages, Vikash et al. successfully isolated intact SCVs under the action of a magnetic field in $2018^{[6]}$. However, unlike macrophages, Salmonella produce Salmonella inducible filaments (SIFs) late in maturation within the epithelium assisting in SCV localization to the perinuclear region near the Golgi ${ }^{[7-8]}$, further limiting the range of activity of Salmonella within the SCV and preventing isolation of SCVs within the epithelium by the same method. Thus, hyper-replication Salmonella within the cytoplasm emerge as another breakthrough in isolating and quantifying the two types of Salmonella within epithelial cells.

Biotin streptavidin amplified enzyme linked immunosorbent assay (BA-ELISA) is an established detection technique based on the principle of conventional ELISA combining the highly amplified effect between biotin and avidin with high sensitivity ${ }^{[9-10]}$. A horseradish peroxidase (HRP)-conjugated avidin molecule reacts with an anti-Salmonella conjugated biotin molecule to act as a multistage amplification ${ }^{[11-12]}$, and HRP reacts with 3,3', $5,5^{\prime}$ - tetramethylbenzidine (TMB) to produce a color change ${ }^{[13]}$, ultimately achieving the goal of quantifying Salmonella. In this study, the concentration of antiSalmonella, anti-Salmonella (biotin), antigen antibody binding time will be optimized to further quantify the amount of Salmonella binding to the antibody. The effective separation and quantification of intracellular free Salmonella from SCV coated Salmonella based on BAELISA, and the improvement of the separation method of host intracellular SCV from free Salmonella will provide a theoretical basis for further related studies to explore the pathogenic mechanism of Salmonella.

\footnotetext{
*E-mail: 781969768@qq.com
} 


\section{Materials and methods}

\subsection{Bacterial strains}

Salmonella enterica serovar Typhimurium LT2 is preserved in the General Microbiology Center of China microbial strain Preservation Management Committee (CGMCC), No.7020.

\subsection{Optimization of the BA-ELISA procedure}

The bottom of the wells of an ELISA plate was coated with a polyclonal rabbit anti-Salmonella antibody(1:50, 1:100, $1: 250,1: 500,1: 1000,1: 2000,1: 400,1: 8000)$ and AntiSalmonella (biotin) antibody( 1:50, 1:100, 1:250, 1:500, $1: 1000,1: 2000,1: 400,1: 8000)$ in PBS and incubated overnight at $4^{\circ} \mathrm{C}$. The optimal concentration of antibody was optimized by using the numbers of Salmonella determined by flat colony counting method. After that, the antibody was co-incubated with quantitative Salmonella at $4^{\circ} \mathrm{C}$ for $0.5 \mathrm{~h}, 1 \mathrm{~h}, 2 \mathrm{~h}$ and $4 \mathrm{~h}$, and the numbers of conjugated Salmonella were used as the intuitive judgment index for optimization.

\subsection{Procedures of BA-ELISA experiment}

The microtiter plate wells were coated with $100 \mu \mathrm{L}$ of the coating antigen(anti-Salmonella antibody) in coating buffer followed by overnight incubation at $4^{\circ} \mathrm{C}$. After four washings with PBST (PBS with $0.05 \%$ Tween-20), Blocking was carried out by incubating the $200 \mu \mathrm{L} /$ wells with a $2 \%$ BSA solution at room temperature for $90 \mathrm{~min}$. The solution was discarded, and the plates were washed, as described above. Then, $100 \mu \mathrm{L}$ of the standard solution were added at $4^{\circ} \mathrm{C}$ for $2 \mathrm{~h}$, after another washing step, 100 $\mu \mathrm{L} /$ well anti-Salmonella (biotin) antibodies were added and incubated at $37^{\circ} \mathrm{C}$ for $1 \mathrm{~h}$. The plates were washed again, $100 \mu \mathrm{L} /$ well of streptavidin-labeled HRP, at a dilution of 1:1000 dilution buffer, was added, and the plates were incubated at $37^{\circ} \mathrm{C}$ for $30 \mathrm{~min}$. The $\mathrm{TMB}$ was added at $100 \mu \mathrm{L} /$ well after a final washing step, and the enzymatic reaction was incubated for $10 \mathrm{~min}$ at $37^{\circ} \mathrm{C}$ and then was terminated by the stop solution $(100 \mu \mathrm{L} /$ well $)$. The absorbance of each well was measured at $450 \mathrm{~nm}$.

\subsection{Cell culture and infection}

Henle-407 cells were cultured in antibiotic-free Dulbecco's modified Eagle medium (DMEM) supplemented with $10 \%$ bovine fetal serum (FBS) at $37^{\circ} \mathrm{C}$ under a $5 \% \mathrm{CO}_{2}$ atmosphere. The cells were digested and passaged with $0.25 \%$ trypsin. Cells at a confluence of $80 \%$ were starved in FBS-deficient DMEM for $2 \mathrm{~h}$. Starved cells were then infected with $S$. Typhimurium at an MOI of 20 for $30 \mathrm{~min}$. After washingthree times with PBS, the infected cells were subsequently incubated with DMEM and $100 \mu \mathrm{g} / \mathrm{mL}$ gentamicin for $30 \mathrm{~min}$. Finally, the infected cells were washed once with prewarmed phosphatebuffered saline (PBS) and then incubated with DMEM and $10 \mu \mathrm{g} / \mathrm{mL}$ gentamicin until the harvest times.

\subsection{Counting of total intracellular Salmonella}

When the cells were infected with Salmonella for $2 \mathrm{~h}, 4 \mathrm{~h}$, $8 \mathrm{~h}$ and $16 \mathrm{~h}$, the cells were washed with PBS for three times and digested by trypsin. The cell precipitates were obtained by $800 \mathrm{~g}$ centrifugation $5 \mathrm{~min}$ at $4^{\circ} \mathrm{C}$. The collected cells were washed three times by ice bath cleavage buffer $(250 \mathrm{mM}$ sucrose, $0.5 \mathrm{mM}$ EGTA, $20 \mathrm{mM}$ HEPES-KOH (pH 7.4), 150mM NaCl, $1 \mathrm{mM}$ PMSF), all steps were performed at $4^{\circ} \mathrm{C}$. Between 30 and 40 strokes were performed, until more than $80 \%$ free nuclei were visible. Nuclei and intact cells were removed by performing three sequential centrifugations, at $100 \mathrm{~g}(1000 \mathrm{rpm})$ for $5 \mathrm{~min}$ each, in order to obtain the PNS. The PNS was divided into two parts, one was used for BAELISA to separate vacuolar Salmonella and cytosolic Salmonella, the other was extracted with a low osmotic solution (250mM sucrose, 0.5mM EGTA, 20mM HEPES$\mathrm{KOH}$ (pH 7.4), 150mM NaCl, 1mM PMSF, $0.2 \%$ Triton $\mathrm{X}-100)$ for secondary extraction, and the total numbers of Salmonella was obtained by flat colony counting method.

\subsection{ELISA for quantification of cytosolic Salmonella and vacuolar Salmonella}

Samples from the PNS were added to the wells and incubated for $2 \mathrm{~h}$ at $4{ }^{\circ} \mathrm{C}$, the experimental procedures were same as 2.3. The vacuolar Salmonella bound in the well by BA-ELISA, and the numbers of total Salmonella, vacuolar Salmonella and cytosolic Salmonella were calculated respectively.

\subsection{Statistical analyses}

Statistical significance was calculated by a one-tailed distributed paired Student $t$ test or by two-way analysis of variance (ANOVA) with Origin 8.6 and SPSS 11.5, results are presented as the means \pm SD for three independent experiments. $p$ values of $<0.05$ were considered to indicate significant differences.

\section{Results}

\subsection{Optimization of the BA-ELISA procedure}

After radiation treatment of highly adsorptive 96-well plate, polystyrene binds to antibody stably through hydrophobic interaction and ion interaction, and the binding of antigen and antibody enables Salmonella antibody to bind to quantitative Salmonella. The numbers of Salmonella bound to antibodies was indirectly obtained by flat colony counting (Fig.1). With the decrease of the dilution ratio of anti- Salmonella antibody and antiSalmonella (biotin) antibody, the numbers of Salmonella bound by antibody increased significantly, which was proportional to the concentration of antibody. With the increase of antibody concentration, the binding amount of Salmonella -antibody reached a certain saturation, the numbers of Salmonella bound by anti-Salmonella antibody was about $3200 \mathrm{CFU}$, while the numbers of 
Salmonella bound by anti-Salmonella (biotin) antibody was about 2700 CFU. Based on the principle of saving antibodies, and in order to ensure the accuracy and feasibility of the experiment, comprehensively considering the needs of the experiment, the concentration of antibody under the dilution of 1:500 as the best concentration for the experiment.

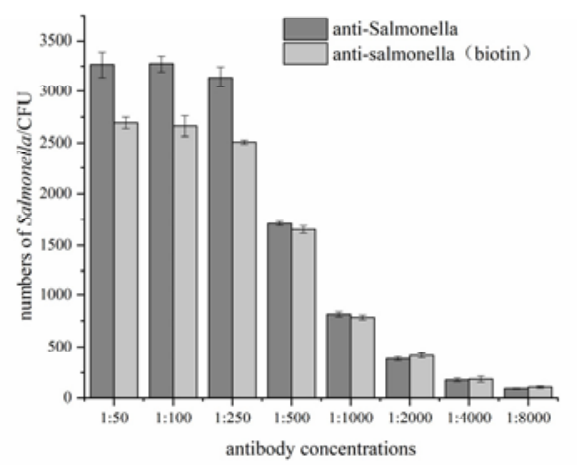

Figure 1. The optimization of anti-Salmonella and antiSalmonella (biotin) antibody concentrations

By comparing the binding amount and binding rate of Salmonella obtained from different incubation time (Table 1), we found that the binding amount of Salmonella increased with the extension of incubation time, but there was no significant difference in the ratio of Salmonella that the antibody could bind between $2 \mathrm{~h}$ and $4 \mathrm{~h}(\mathrm{p}<0.05)$. Therefore, the best incubation time for Salmonella to bind with antibody at $4^{\circ} \mathrm{C}$ is $2 \mathrm{~h}$.

Table1. effect of incubation time on the ability of antigenantibody binding

\begin{tabular}{cccc}
\hline $\begin{array}{c}\text { Incubation } \\
\text { time } / \mathrm{h}\end{array}$ & Total bacteria count & $\begin{array}{c}\text { Binding } \\
\text { capacity }\end{array}$ & Binding rate \\
\hline 0.5 & $459 \pm 46.34$ & $134 \pm 24.74$ & $29.12 \% \pm$ \\
& & & $0.62 \%^{\mathrm{a}}$ \\
1 & $714 \pm 68.94$ & $269 \pm 50.72$ & $37.68 \% \pm$ \\
& & & $1.18 \% \mathrm{o}$ \\
2 & $1232 \pm 38.08$ & $862 \pm 79.15$ & $69.96 \% \pm$ \\
& & & $0.78 \%{ }^{\mathrm{c}}$ \\
4 & $903 \pm 84.42$ & $633 \pm 54.44$ & $70.08 \% \pm$ \\
& & & $1.01 \%^{\mathrm{c}}$ \\
\hline
\end{tabular}

Note: different lowercase letters indicate significant differences between groups $(\mathrm{p}<0.05)$.

\subsection{Binding amount and binding rate of anti- Salmonella antibody to Salmonella}

The numbers of Salmonella in the well was 2000-3500 CFU, and the Salmonella bound by anti-Salmonella antibody reached saturation at $\sim 1700$ (Fig.2A). The binding capacity did not increase with the the numbers of bacteria added in the well, but showed a stable binding capacity. On the premise that there is a certain binding amount of Salmonella and anti-Salmonella antibody, it is necessary to further clarify the binding efficiency of Salmonella and anti-Salmonella antibody. Therefore, we reduce the numbers of Salmonella in the well to reduce the systematic error. When the numbers of Salmonella in the well lower than the maximum binding amount of Salmonella, the binding rate can't up to $100 \%$, the numbers of salmonella bound with anti-Salmonella was floating, and the binding rate was stable at about $70 \%$ (Fig.2B).
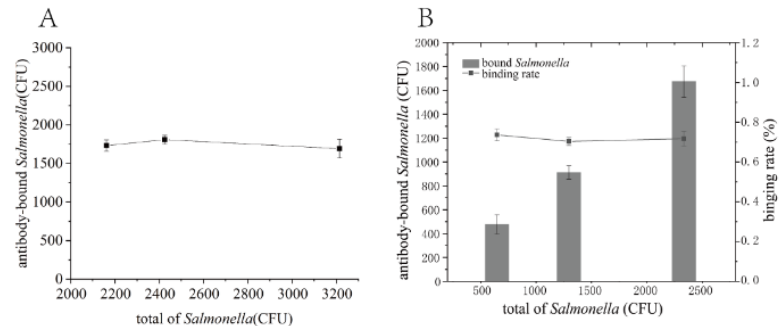

Figure 2. Salmonella binding capacity (A) and binding rate (B) with anti-Salmonella antibody

\subsection{Construction of Salmonella-absorbance standard curve by BA-ELISA}

The construction of Salmonella -absorbance standard curve for quantitative cytosolic Salmonella is essential for the isolation of vacuolar Salmonella and cytosolic Salmonella by BA-ELISA. The anti-Salmonella antibody in the well binds to the cytosolic Salmonella, while the SCV does not bind to the anti-Salmonella antibody, so it is necessary to quantify the unbound Salmonella by plate counting. The Salmonella bound antibody can be quantified by $\mathrm{OD}_{450 \mathrm{~nm}}$ value (Fig.3A and Fig.3B). There is no linear and exponential relationship between numbers of Salmonella and $\mathrm{OD}_{450 \mathrm{~nm}}$ value, but there is a linear positive correlation between $\lg ^{\text {(number }}$ of Salmonella) and $1 \mathrm{~g}^{\mathrm{OD} 450 \mathrm{~nm}}$, which can quickly and accurately calculate the number of Salmonella in the well.
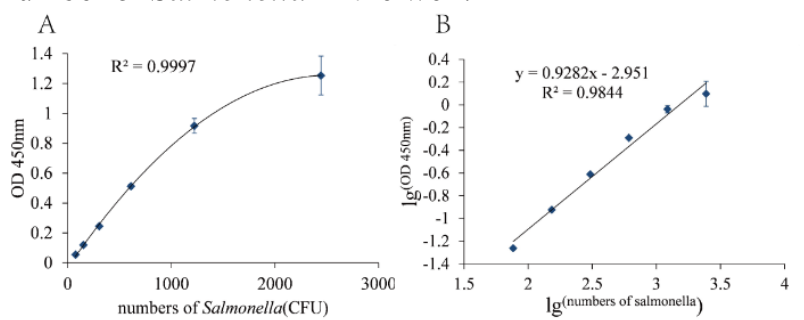

Figure 3. Construction of Salmonella-Absorbance Standard Curve by BA-ELISA

\subsection{Isolation of intracellular cytosolic Salmonella and vacuolar Salmonella}

The total of intracellular bacteria, cytosolic Salmonella and vacuolar Salmonella were counted by BA-ELISA and flat colony counting, the total of intracellular bacteria proliferated slowly within $4 \mathrm{~h}$ of Salmonella infection (Fig.4A). In the early stage of infection, most Salmonella survived in SCV and proliferated at a moderate rate or survived in a dormant state ${ }^{[14]}$. The proportion of numbers of vacuolar Salmonella was negatively correlated during Salmonella infection, resulting in only about $23 \%$ of vacuolar Salmonella (16h). The proliferation rate was significantly lower than cytosolic Salmonella. With the prolongation of infection time, the Salmonella escaped from SCV and propagated rapidly in the cytoplasm. The numbers of cytosolic Salmonella in the cytoplasm exceeded the numbers of vacuolar Salmonella at $8 \mathrm{~h}$ after Salmonella infection, and far exceeded the vacuolar Salmonella at $16 \mathrm{~h}$, and the proportion in the cell gradually increased (Fig.4B), the proportion of cytosolic Salmonella and vacuolar Salmonella was about $22 \%$ and $70 \%$ after 
Salmonella infection (2h), and the proportion of cytosolic Salmonella in the host cell was positively correlated during Salmonella infection, reaching about 65\% (16h).
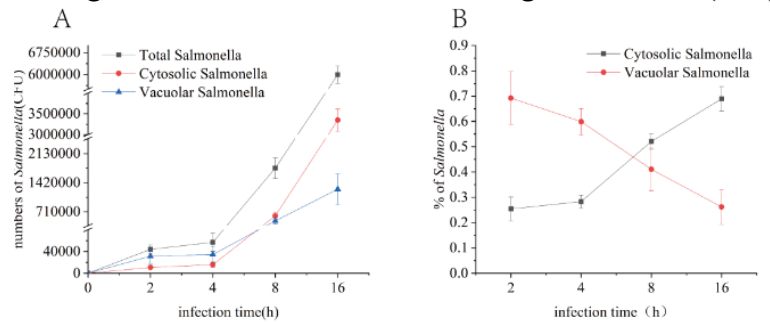

Figure 4. A Dynamic growth curve of Salmonella in Henle407 cells; B Relative percentage of cytosolic Salmonella and vacuolar Salmonella in Henle-407 cells

\section{Conclusion}

Salmonella enterica serovar Typhimurium can inject effector proteins into host cells via type III secretion systems (T3SSs), the effector induces the recombination of cytoskeleton proteins and formation of ruffles in the host cell membrane, establishing a replicative niche termed SCV ${ }^{[15-16]}$, which permits $S$. Typhimurium survive and reproduce within SCV. Meanwhile, part of Salmonella escape from SCV leading to the robust replication within cytosol, cytosolic Salmonella and vacuolar Salmonella coexisted in the host cells ${ }^{[17]}$. But there are significant differences in intracellular sublocalization and reproduction rate between the two forms of Salmonella, the current researches on the mechanism of Salmonella infection are mostly based on the total numbers, and there is no clear distinction between cytosolic Salmonella and vacuolar Salmonella.

In this study, we use BA-ELISA combined with flat colony counting methods to isolate and quantify intracellular cytosolic Salmonella and vacuolar Salmonella, so as to obtain a more intuitive reproductive trend of cytosolic Salmonella and vacuolar Salmonella. First, we determined the antibody concentration and antigen-antibody binding time. Second, through the antigen-antibody specific binding based on BA-ELISA, we confirmed the quantitative binding of anti-Salmonella antibody to about 1700 of Salmonella. Unlike protein, Salmonella is always in a state of movement within buffer. Although under the influence of gravity, Salmonella can bind to antibody, this effect has a certain relationship with time and temperature, resulting in that the binding rate between Salmonella and antibody is only $70 \%$. Third, intracellular Salmonella was obtained by breaking cells in a homogenizer. With the help of homogenized buffer, this mild fragmentation method not only released intracellular Salmonella but also minimized the damage to SCV. Based on the binding capacity and binding rate, the numbers of cytosolic Salmonella were quantified by BA-ELISA, so far, the vacuolar Salmonella and cytosolic Salmonella were isolated and quantified in a direct and clear way. It is more intuitive and accurate to obtain the dynamic reproduction trend of two forms of Salmonella in the host cells. This method improves the counting way of intracellular Salmonella and provides a theoretical basis for the study of the mechanism of Salmonella infection.

\section{References}

1. Tang BL. Bacteria-Containing Vacuoles: Subversion of Cellular Membrane Traffic and Autophagy. J. Critical Reviews in Eukaryotic Gene Expression, 25(2):163-174(2015)

2. McQuate SE, Young AM, Silva-Herzog E, et al. Long-term live-cell imaging reveals new roles for Salmonella effector proteins SseG and SteA. J. Cellular Microbiology, 19(1):10 (2017)

3. Steele-Mortimer O, Méresse S, Gorvel JP, et al. Biogenesis of Salmonella typhimurium-containing vacuoles in epithelial cells involves interactions with the early endocytic pathway. J. Cellular Microbiology, 1(1):33-49(2010)

4. Knodler LA. Salmonella enterica: living a double life in epithelial cells. J. Current Opinion in Microbiology, 23:23-31(2015)

5. Lührmann A, Haas A. A method to purify bacteriacontaining phagosomes from infected macrophages. $\mathrm{J}$. Methods in Cell Science, 22(4):329-341(2000)

6. Singh V, Schwerk P, Tedin K. Rapid Isolation of intact Salmonella-containing vacuoles using paramagnetic nanoparticles. J. Gut Pathogens, 10(1):33(2018)

7. Santos JC, Duchateau M, Fredlund J, et al. The COPII complex and lysosomal VAMP7 determine intracellular Salmonella localization and growth. J. Cell Microbiology. 17(12):1699-1720(2015)

8. Liss V, Swart AL, Kehl A, et al. Salmonella enterica Remodels the Host Cell Endosomal System for Efficient Intravacuolar Nutrition. J. Cell Host \& Microbe, 21(3):390-402(2017)

9. Cui X, Vasylieva N, Shen D, et al. Biotinylated single-chain variable fragment-based enzyme-linked immunosorbent assay for glycocholic acid. J. Analyst. 143(9):2057-2065(2018)

10. Sun Z, Lv J, Liu X, et al. Development of a NanobodyAviTag Fusion Protein and Its Application in a Streptavidin-Biotin-Amplified Enzyme-Linked Immunosorbent Assay for Ochratoxin A in Cereal. J. Analytical Chemistry. 90(17):10628-10634(2018)

11. Sedlak SM, Schendel LC, Melo MCR, et al. Direction Matters: Monovalent Streptavidin/Biotin Complex under Load. J. Nano Letters. 19(6):3415-3421(2019)

12. Sun Z, Wang X, Tang Z, et al. Development of a biotin-streptavidin-amplified nanobody-based ELISA for ochratoxin $A$ in cereal. J. Ecotoxicology \& Environmental Safety. 171:382-388(2019)

13. Zhang X, Yang Q, Lang Y, et al. Rationale of 3,3',5,5'Tetramethylbenzidine as the Chromogenic Substrate in Colorimetric Analysis. J. Analytical Chemistry. 92(18):12400-12406(2020)

14. Röder J, Hensel M. Presence of SopE and mode of infection result in increased Salmonella-containing vacuole damage and cytosolic release during host cell infection by Salmonella enterica. J. Cellular Microbiology, 22(5):e13155(2020) 
15. Kaiyi Z, Ambre R, Monika N, et al. Minimal SPI1T3SS effector requirement for Salmonella enterocyte invasion and intracellular proliferation in vivo. $\mathrm{J}$. Plos Pathogens, 14(3):e1006925(2018)

16. Finn $\mathrm{CE}$, Chong A, Cooper $\mathrm{KG}$, et al. A second wave of Salmonella T3SS1 activity prolongs the lifespan of infected epithelial cells. J. PLoS Pathogens. 13(4):e1006354(2017)

17. Wu S, Shen Y, Zhang S, et al. Salmonella Interacts With Autophagy to Offense or Defense. J. Frontiers in Microbiology. 11:721(2020) 